TRANSACTIONS OF THE

AMERICAN MATHEMATICAL SOCIETY

Volume 275, Number 1, January 1983

\title{
LOCALLY STRANGE HYPERBOLIC SETS
}

BY

LOWELL JONES

\begin{abstract}
ABSTRACr. The purpose of this paper is to present a very general method of constructing basic sets having complicated local homeomorphism types.
\end{abstract}

Introduction. Recall that a hyperbolic set for a diffeomorphism $f$ of a smooth manifold $M$ is a compact subset $\Lambda$, contained in the interior of $M$, and satisfying

(a) $f: \Lambda \rightarrow \Lambda$ is a homeomorphism;

(b) the tangent bundle of $M$ splits over $\Lambda,\left.T(M)\right|_{\Lambda}=\xi_{u}+\xi_{s}$, as a Whithey sum of continuous subbundles each of which is left invariant by $d f$ (the differential of $f$ );

(c) for some Riemannian metric || on $M,|d f(v)|>|v|$ for each nonzero $v \in \xi_{u}$, and $|d f(v)|<|v|$ for each nonzero $v \in \xi_{s}$.

The hyperbolic set $\Lambda$ is a basic set for $f: M \rightarrow M$ provided the periodic points of $\left.f\right|_{\Lambda}$ are dense in $\Lambda,\left.f\right|_{\Lambda}$ has a dense orbit, and there is a compact neighborhood $U$ for $\Lambda$ in $M$ such that $\bigcap_{n=-\infty}^{\infty} f^{n}(U)=\Lambda$.

In $[2,3]$ F. T. Farrell and the author showed that the typological type of a basic set can vary enormously. In this paper it will be shown that the local topological type of a basic set can also vary enormously.

Concerning these local topological types, some facts have already been discovered. Williams [9, Theorem C] showed that every expanding attractor, whose stable foliation is $C^{1}$, is locally homeomorphic to the product of the Cantor set and Euclidean space. The restriction on the stable foliation was later removed by Robinson and Williams [7]. On the other hand, Guckenheimer [4, §6] has constructed examples of one-dimensional basic sets which are not locally homeomorphic to the product of any closed subset of the Cantor set with the line. Still, all examples of basic sets known to date have the property that their local homotopy groups vanish in dimensions above one.

The examples of basic sets constructed in this paper achieve a wide variety of local homotopy types, in particular, nonvanishing local homotopy groups may appear in any dimension (see 1.6 and 3.1). The most interesting of these examples are those which are locally homeomorphic to an $l$-dimensional Cantor set crossed with the (zero-dimensional) Cantor set. An l-dimensional Cantor set is any closed subset $C_{l}$ of the $(l+1)$-dimensional ball $B^{l+1}$, satisfying

0.0 . (a) $\overline{B^{l+1}-C_{l}}$ is the disjoint union of a countable collection of closed $(l+1)$ dimensional topological balls, $B_{1}, B_{2}, B_{3}, \ldots$;

Received by the editors August 17, 1981 and, in revised form, November 16, 1981.

1980 Mathematics Subject Classification. Primary 58F15; Secondary 57R50. 
(b) $\lim _{i \rightarrow \infty}\left[\operatorname{diameter}\left(B_{i}\right)\right]=0$;

(c) the $\left\{B_{i}\right\}$ are a dense collection of closed balls in $B^{l+1}$.

The topological dimension of $C_{l}$ is equal to $l$ (see 4.1).

The main result of this paper is the construction of basic sets $\Lambda_{l}$, for each $l>0$, satisfying

0.1 . (a) Every $p \in \Lambda$, has a neighborhood $V$ which is the cartesian product of the (zero-dimensional) Cantor set with a space $U$.

(b) $U$ is homeomorphic to an open subset in an $l$-dimensional Cantor set $C_{l}$.

Such a basic set is the result of Theorems 1.6 and 3.1. Basic sets, achieving much more complicated local topological types than the $\Lambda_{l}$, can also be constructed by the same techniques which yield the $\Lambda_{l}$ (see 3.7).

All the basic sets of this paper are relative l-solenoids. This concept, which generalizes William's $n$-solenoid idea (see [9]) is described in $\$ 1$. Every relative $l$-solenoid is a basic set (see 1.6). In $\S 3$ the relative $l$-solenoids $\Lambda_{l}$ are constructed. Their construction requires techniques developed by F. T. Farrell and the author in [3].

1. In this section relative $l$-solenoids are discussed. It is proven that every $l$-solenoid is a basic set.

DEFINITION 1.1. By a compact smooth nonsingular branched $n$-manifold with boundary is meant a compact metrizable space $K$ with closed subspace $\partial K$, together with finite collections of closed subsets $\left\{U_{i}\right\}$ and $\left\{D_{i j}\right\}$, and mappings $\left\{\pi_{i}: U_{i} \rightarrow X_{i}\right\}$, subject to the following conditions (see Figure 1.2):

(a) $\cup_{j} D_{i j}=U_{i}, \cup_{i}$ Interior $\left(U_{i}\right)=K$.

(b) Let $q>0$ be the minimum integer such that $U_{i} \cap \partial K=\varnothing$ for all $i \leqslant q$. Then for $i \leqslant q, X_{i}$ is a smooth $n$-ball in $R^{n}$ (i.e., $X_{i}$ is the image of $\left\{x \in R^{n}|| x \mid \leqslant 1\right\}$ under a smooth diffeomorphism $R^{n} \rightarrow R^{n}$ ). For $i>q, X_{i}$ is a smooth half $n$-ball in $R^{n}$ (i.e., $X_{i}$ is the image of $\left\{x \in R^{n}|| x \mid \leqslant 1\right\}, x_{n} \geqslant 0$ under a smooth diffeomorphism $\mathbf{R}^{n} \rightarrow \mathbf{R}^{n}$ which maps $\mathbf{R}^{n-1} \rightarrow \mathbf{R}^{n-1}$ ).

(c) For each $i>q$ let $X_{i}^{\prime}$ denote the subset of $X_{i}$ corresponding to the $(n-1)$-ball $\left\{x \in R^{n}|| x \mid \leqslant 1, x_{n}=0\right\}$. Then $\partial K \equiv \cup_{i>q} \pi_{i}^{-1}\left(X_{i}^{\prime}\right)$.

(d) Each $\pi_{i}: D_{i j} \rightarrow X_{i}$ is a homeomorphism.

(e) There is a cocycle of smooth diffeomorphisms

$$
\alpha_{k l}: \pi_{l}\left(U_{l} \cap U_{k}\right) \rightarrow \pi_{k}\left(U_{l} \cap U_{k}\right) \text { such that } \pi_{k}=\alpha_{k l} \circ \pi_{l}
$$

The subset $\partial K$, together with the subsets $\left\{U_{i} \cap \partial K\right\},\left\{D_{i j} \cap \partial K\right\}$, and the maps $\left\{\pi_{i}: U_{i} \cap \partial K \rightarrow X_{i}^{\prime}\right\}, \quad\left\{\alpha_{k l}: \pi_{l}\left(U_{l} \cap U_{k} \cap \partial K\right) \rightarrow \pi_{k}\left(U_{l} \cap U_{k} \cap \partial K\right)\right\}$ constitute a smooth nonsingular branched (n-1)-manifold, called the boundary of the branched manifold $K$. Note the boundary of $\partial K$ is empty.

A mapping $h: K \rightarrow K^{\prime}$ between two smooth nonsingular branched manifolds is a smooth mapping if all of the compositions

$$
\pi_{j}\left(D_{i j}\right) \stackrel{\pi_{j}^{-1}}{\rightarrow} D_{i j} \stackrel{h}{\rightarrow} U_{i^{\prime}}^{\prime} \stackrel{\pi_{i^{\prime}}^{\prime}}{\rightarrow} \pi_{i^{\prime}}^{\prime}\left(U_{i^{\prime}}^{\prime}\right)
$$

are smooth, where defined. 


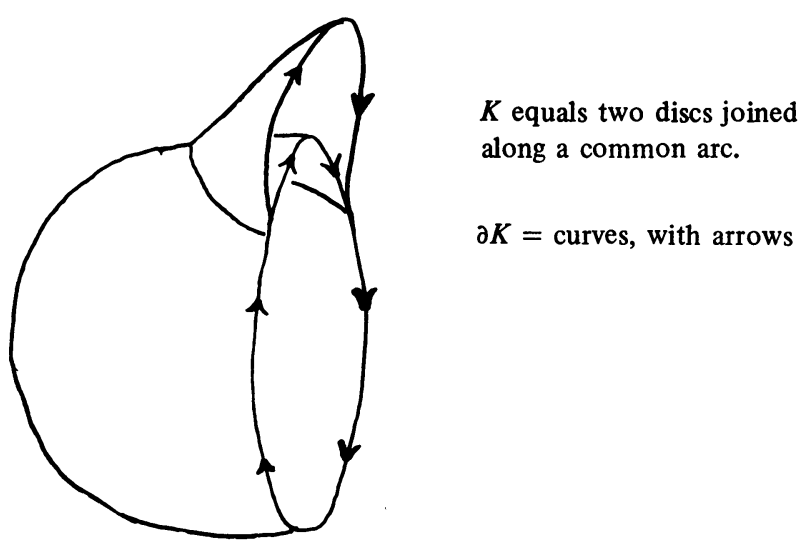

Figure 1.2

A smooth nonsingular branched manifold $K$ with boundary $\partial K$ has a tangent bundle, $T(K)$, defined as follows: Glue all the linear bundles $U_{i} \times R^{n} \rightarrow U_{i}$ together along the linear isomorphisms

$$
1 \times d \alpha_{i j}:\left(U_{i} \cap U_{j}\right) \times R^{n} \rightarrow\left(U_{i} \cap U_{j}\right) \times R^{n} .
$$

The cocyle condition on the $\alpha_{i j}(1.1(\mathrm{e}))$ assures that we get an $n$-dimensional vector bundle $T(K)$ over $K$. Note

$$
T(\partial K)+I=\left.T(K)\right|_{\partial K} .
$$

Any smooth map $h: K \rightarrow K^{\prime}$ induces a bundle map $d h: T(K) \rightarrow T\left(K^{\prime}\right)$ defined by $\left.d h\right|_{X} \equiv d\left(\pi_{i^{\prime}}^{\prime} \circ h \circ \pi_{i}^{-1}\right)$, where

$$
X=\left.T(K)\right|_{\left(D_{i j} \cap h^{-1}\left(U_{i^{\prime}}^{\prime}\right)\right)} .
$$

A smooth mapping $h: K \rightarrow K^{\prime}$ is an immersion if $d h: T(K) \rightarrow T\left(K^{\prime}\right)$ is a linear monomorphism on each fiber of $T(K)$.

DEFINITION 1.3. A smooth nonsingular branched submanifold of codimension zero in $K$ is any subset $W \subset K-\partial K$ of the form $W=f^{-1}(-\infty, 0]$, where $f: K \rightarrow R$ is a smooth map to the real numbers such that the bundle map $d f: T(K) \rightarrow T(R)$ is of rank 1 on each fiber over $f^{-1}(0)$. Note that $W$ is a smooth nonsingular branched manifold with boundary $\partial W \equiv f^{-1}(0)$.

We shall consider smooth immersions $h: W \rightarrow K$ satisfying

Axioms 1.4. (a) $W$ is a smooth nonsingular branched submanifold of codimension zero in the smooth nonsingular branched $n$-manifold $K$ with boundary $\partial K$.

(b) $h(\partial W) \subset \partial K$.

(c) There are $a>0, \lambda>1$, and a Reimannian metric \| on the fibers of $T(K)$, so that $\left|d h^{n}(v)\right|>a \lambda^{n}|v|$ holds for any $v \in T(W)$ whenever $d h^{n}(v)$ is defined.

(d) Set $\Lambda_{j} \equiv\left\{x \in W \mid h^{i}(x) \in W \forall 0 \leqslant i \leqslant j\right\}$. Set $\Lambda \equiv \bigcap_{j} \Lambda_{j}$. For any $x \in \Lambda$ and any smooth $n$-ball, $B$, containing $x$ in its interior, there is $j>0$ so that $h^{j}$ maps a subset of $B$ onto $W$.

(e) For each $x \in W-\partial W$ there is a neighborhood $U$ for $x$ in $W$ such that $h(U)$ is contained in a smooth $n$-ball of $K$. 
Following Williams [9], we now set $\Sigma(h)$ equal to the inverse limit of $\Lambda^{h} \stackrel{h}{\leftarrow} \Lambda^{h}$ $\Lambda \stackrel{h}{\leftarrow} \Lambda \stackrel{h}{\leftarrow} \cdots$, and define $H: \Sigma(h) \rightarrow \Sigma(h)$ to be the shift

$$
\begin{aligned}
H\left(x_{0}, x_{1}, x_{2}, x_{3}, \ldots\right) & \equiv\left(h\left(x_{0}\right), h\left(x_{1}\right), h\left(x_{2}\right), \ldots\right) \\
& =\left(h\left(x_{0}\right), x_{0}, x_{1}, x_{2}, \ldots\right) .
\end{aligned}
$$

Definition 1.5. Suppose dimension $(\Sigma(h))=l$. Then $\Sigma(f)$ is called a relative l-solenoid and $H: \Sigma(h) \rightarrow \Sigma(h)$ is the shift map.

THEOREM 1.6. Any relative solenoid $H: \Sigma(h) \rightarrow \Sigma(h)$ is topologically conjugate to a basic set with map.

Proof OF 1.6. The proof of 1.6 is very similar to the proof of Theorem B in [9], so I will only sketch it.

We begin with a few definitions. Let $h: W \rightarrow K, h^{\prime}: W^{\prime} \rightarrow K^{\prime}$ denote two maps satisfying 1.4, and $\Lambda, \Lambda^{\prime}$ the subsets of $W, W^{\prime}$ given in 1.4(d). The maps $h, h^{\prime}$ are shift equivalent if there are open neighborhoods $U, U^{\prime}$ for $\Lambda, \Lambda^{\prime}$ in $W, W^{\prime}$, smooth immersions $r: U \rightarrow W^{\prime}, s: U^{\prime} \rightarrow W$, and a positive integer $m$ so that $h s=s h^{\prime}$, $h^{\prime} r=r h$, and $s r=h^{m}, r s=\left(h^{\prime}\right)^{m}$ hold wherever the compositions are defined.

A smooth nonsingular branched manifold with boundary, $W$, is said to be normally branched if $W \subset K$ where $K$ is as in 1.4 and $\partial K=\varnothing$ and $K$ is normally branched as described in 5.5 of [9].

The first step of this proof is to replace $h: W \rightarrow K$ by a shift equivalent map $h^{\prime}$ : $W^{\prime} \rightarrow K^{\prime}$ such that $K^{\prime}$ is normally branched.

This is accomplished as in Lemma 5.6 of [9].

The next step in the proof is to show that the two shifts $H: \Sigma(h) \rightarrow \Sigma(h)$ and $H^{\prime}$ : $\Sigma\left(h^{\prime}\right) \rightarrow \Sigma\left(h^{\prime}\right)$, associated to $h: W \rightarrow K$ and $h^{\prime}: W^{\prime} \rightarrow K^{\prime}$ respectively, are topologically conjugate. This is accomplished as in Theorem 5.2 of [9].

The next step of this proof is to construct a diffeomorphism $g: U \rightarrow R^{m}$ from an open subset of $R^{m}$ into $R^{m}$ so that $U$ contains a compact subset $\Omega$ satisfying

1.7.(a) $g: \Omega \rightarrow \Omega$ is a homeomorphism topologically conjugate to $H^{\prime}: \Sigma\left(h^{\prime}\right) \rightarrow \Sigma\left(h^{\prime}\right)$.

(b) $\Omega=\left\{x \in U \mid g^{i}(x) \in U\right.$ for all $\left.i \in Z\right\}$.

(c) $\Omega$ is a hyperbolic subset of $g: U \rightarrow R^{m}$.This is accomplished as in $\S 8$ of [9].

To complete the proof of 1.6 it suffices to show that $g: \Omega \rightarrow \Omega$ of 1.7 has a dense orbit and that the periodic points of $g$ are dense in $\Omega$ (see 9.2 in [8]).

I will first show that $g: \Omega \rightarrow \Omega$ has a dense orbit by showing that $H^{\prime}: \Sigma\left(h^{\prime}\right) \rightarrow \Sigma\left(h^{\prime}\right)$ has a dense orbit (see 1.7(a)). Any point in $\Sigma\left(h^{\prime}\right)$ has the form $\left(x_{0}, x_{1}, x_{2}, \ldots\right)$ where $h\left(x_{i}\right)=x_{i-1}$. Note that the $H^{\prime}$-orbit of $\left(x_{0}, x_{1}, x_{2}, x_{3}, \ldots\right)$ will be dense in $\Sigma\left(h^{\prime}\right)$ if the $h^{\prime}$-orbit of $x_{0}$ is dense in $\Lambda^{\prime}$. To find such an $x_{0} \in \Lambda^{\prime}$, first choose a dense sequence in $\Lambda^{\prime}, y_{1}, y_{2}, y_{3}, \ldots$ By using $1.4(\mathrm{~d})$ over and over, a nested sequence of smooth $n$-balls $B_{1} \supset B_{2} \supset B_{3} \supset \cdots$ can be constructed in $W^{\prime}$ satisfying

(a) $\lim _{i \rightarrow \infty} \operatorname{diameter}\left(B_{i}\right)=0$;

(b) for some positive integer $\phi(i), f^{\phi(i)}\left(B_{i}\right)$ contains $y_{i}$ in its interior and is contained in a neighborhood $U_{i}$ of $y_{i}$ with diameter $\left(U_{i}\right)<\left(\frac{1}{2}\right)^{i}$.

Set $x_{0} \equiv \bigcap_{i} B_{i}$. Then by (a), (b) directly above, and the choice of the $\left\{y_{i}\right\}, x_{0}$ is a point of $\Lambda^{\prime}$ whose $h$-orbit is dense in $\Lambda^{\prime}$. 
Finally, I will show that the periodic points of $g: \Omega \rightarrow \Omega$ are dense by showing that those of $H^{\prime}: \Sigma\left(h^{\prime}\right) \rightarrow \Sigma\left(h^{\prime}\right)$ and dense (see 1.7(a)).

Since $\Sigma\left(h^{\prime}\right)$ is the inverse limit of

$$
\Lambda^{\prime} \stackrel{h^{\prime}}{\leftarrow} \Lambda^{\prime} \stackrel{h^{\prime}}{\leftarrow} \Lambda^{\prime} \stackrel{h^{\prime}}{\leftarrow \cdots}
$$

any point in $\Sigma\left(h^{\prime}\right)$ has the form $\left(x_{0}, x_{1}, x_{2}, \ldots\right)$ with $x_{i} \in \Lambda^{\prime}$ and $h^{\prime}\left(x_{i}\right)=x_{i-1}$. Using $1.4(\mathrm{~d})$ and the fact that $W^{\prime}$ is normally branched, one can choose, for each $i>0$, a smooth $n$-ball, $B$, in $W$ containing $x_{i}$ on its interior and having arbitrarily small diameter $\varepsilon_{i}$, so that for some $j>0$ and some ball $B^{\prime} \subset B, h^{j}: B^{\prime} \rightarrow B$ is a diffeomorphism. Let $x \in B^{\prime}$ denote the fixed point of $h^{j}: B^{\prime} \rightarrow B$. Let $p_{i} \in \Sigma\left(h^{\prime}\right)$ denote the point $\left(x_{0}^{\prime}, x_{1}^{\prime}, x_{2}^{\prime}, \ldots\right)$ which is a periodic point of period $j$ for $H^{\prime}$ : $\Sigma\left(h^{\prime}\right) \rightarrow \Sigma\left(h^{\prime}\right)$ and satisfies $x_{i+k}^{\prime} \equiv h^{j-k}(x)$ for $0 \leqslant k \leqslant j$. The sequence $\left\{p_{i}\right\}$ converges to $\left(x_{0}, x_{1}, x_{2}, \ldots\right)$ provided each $\varepsilon_{i}$ is chosen sufficiently small. This completes the proof of 1.6 .

2. The main concern of this paper is the construction of relative $l$-solenoids which have strange local homeomorphism types. In this section a result is proven that relates the local homeomorphism type of $\Sigma(h)$ to that of $\Lambda$, where $\Lambda, \Sigma(h)$ are as in $1.4,1.5$.

Proposition 2.1. Let $h: W \rightarrow K, \Lambda$, and $H: \Sigma(h) \rightarrow \Sigma(h)$ be as in 1.4, 1.5 . Suppose $K$ is normally branched. Then for any $y \in \Sigma(h)$ there is a smooth n-dimensional ball $B$ in $K$ so that the cartesian product of $\Lambda \cap(B-\partial B)$ with the Cantor set is homeomorphic to a neighborhood for $y$ in $\Sigma(h)$. Here $\partial B$ is the manifold boundary of $B$.

Proof of 2.1. Since $K$ is normally branched it follows that the $U_{i}$ and $D_{i, j}$ in 1.1 can be chosen to satisfy the following:

2.2.(a) The topological boundary of $U_{i}$ equals $\cup_{j} \partial D_{i, j}$ if $U_{i} \subset K-\partial K$.

(b) If $Y$ is a smooth $n$-dimensional submanifold of $K$ contained in some $U_{i}$, then $Y$ is contained in one of the $D_{i, j}$. Moreover, no one of the $D_{i, j}$ is contained in the union of the other $D_{i, j}$. In the rest of this proof $N$ will denote the number of distinct $D_{i j}$.

In this proof $U$ shall denote any open set in $K$ whose closure, $\bar{U}$, satisfies

2.3.(a) $\bar{U}$ is a union $\cup_{i} B_{i}$ of at most $N$ distinct smooth $n$-dimensional balls $B_{i}$, where $N$ comes from 2.2 .

(b) Each $B_{i}$ is mapped by $h: W \rightarrow K$ diffeomorphically onto the same smooth ball in $K$.

(c) If $Y$ is a connected smooth $n$-dimensional manifold in $\bar{U}$, then $Y \subset B_{i}$ for some $i$.

Recall $\Sigma(h)$ is the inverse limit of $\Lambda \stackrel{h}{\leftarrow} \Lambda \stackrel{h}{\leftarrow} \Lambda \stackrel{h}{\leftarrow} \cdots$. Let $\rho: \Sigma(h) \rightarrow \Lambda$ denote the projection, i.e. $\rho\left(x_{0}, x_{1}, x_{2}, \ldots\right) \equiv x_{0}$ for any $\left(x_{0}, x_{1}, \ldots\right)$ in the inverse limit space $\Sigma(h)$. Denote by $V$ any open set in $\Sigma(h)$ satisfying

2.4.(a) $V \equiv \rho^{-1}(U \cap \Lambda)$, where $U$ is as in 2.3. The proof of 2.1 will be completed by showing that for any $y \in \Sigma(h)$ there is $V$ as in 2.4(a) containing $y$ and satisfying

(b) $V$ is a disjoint union $\cup_{i} V_{i}$ of at most $N$ open sets $V_{i}$, where $N$ comes from 2.2. 
(c) Each $V_{i}$ is homeomorphic to the cartesian product of the Cantor set with $\left(B_{i}-\partial B_{i}\right) \cap \Lambda$, where $B_{i}$ is one of the balls in 2.3(a).

To verify 2.4 the following lemma is needed. For any $U$ as in 2.3 denote by $S_{k, 1}$, $S_{k, 2}, \ldots$ the different path connected components of $h^{-k}(U)$.

Lemma 2.5. There is $\varepsilon>0$ so that if diameter $(U)<\varepsilon$ and $U \cap \Lambda \neq \varnothing$ then $S_{k, i}$ satisfy the following:

(a) There is a finite number of the $S_{k, i}$ for each fixed $k$.

(b) Each closure $\bar{S}_{k, j}$ is mapped onto one of the balls $B_{i}$ in 2.3(a) by $h^{k}$.

(c) Each closure $\bar{S}_{k, j}$ satisfies $2.3(\mathrm{a})-(\mathrm{c})$.

First 2.4 will be verified, using 2.5 , and then 2.5 will be proven.

For $y \in \Sigma(h)$, write $y=\left(y_{0}, y_{1}, y_{2}, \ldots\right)$, where $y_{j} \in \Lambda$ for all $j \geqslant 0$. Choose a neighborhood $U$ for $y_{0}$ in $K$ such that diameter $(U)<\varepsilon$, where $\varepsilon$ comes from 2.5 and $\bar{U}$ satisfies 2.3. It follows from 1.4(e), 2.2 that such a neighborhood exists. Define $V_{i}$ in $2.4(\mathrm{~b})$ to be all $\left(x_{0}, x_{1}, x_{2}, \ldots\right)$ in $V$ such that, for all $k \geqslant 0, x_{k} \in S_{k, j}$ with $h^{k}\left(S_{k, j}\right)=B_{i}$ (see $\left.2.5(\mathrm{~b})\right)$. The $V_{i}$ are disjoint because the $\left\{S_{k, 1}, S_{k, 2}, \ldots\right\}$ are disjoint and the $B_{i}$ are distinct. If $\left(Z_{0}, Z_{1}, \ldots\right)$ is in $V$, then for all $k$, we have $Z_{k} \in S_{k, l}$ for some $l$ dependent on $k$. Since $H\left(Z_{k}\right)=Z_{k-1}$, we have $h\left(S_{k, l}\right) \subset S_{k-1, l^{\prime}}$, where $Z_{k-1} \in S_{k-1, l^{\prime}}$. Hence $h^{k}\left(S_{k, l^{\prime}}\right)=h^{k-1}\left(S_{k-1, l^{\prime}}\right)$ for all $k>0$ (see $2.5(\mathrm{~b})$ ), so $\left(Z_{0}, Z_{1}, \ldots\right)$ must be in one of the $V_{i}$. This completes the verification of 2.4(b).

To verify $2.4(\mathrm{c})$, I will first define a continuous map $r: V_{i} \rightarrow Q$ into a closed zero-dimensional subset of $[0,1]$. It will then be proven that $Q$ is homeomorphic to the Cantor set, and that $\rho \times r: V_{i} \rightarrow \Lambda \cap\left(B_{i}-\partial B_{i}\right) \times Q$ is a homeomorphism.

To each $S_{k, j}$, satisfying $h^{k}\left(\bar{S}_{k, j}\right)=B_{i}($ see $2.5(\mathrm{~b}))$, associate a closed interval $I_{k, j}$ in $[0,1]$, satisfying

2.6.(a) $I_{k+1, j} \subset I_{k, i}$ iff $h\left(S_{k+1, j}\right) \subset S_{k, i}$.

(b) The $I_{k, 1}, I_{k, 2}, \ldots$ are disjoint.

(c) limit $_{k \rightarrow \infty}\left[\operatorname{diameter}\left(I_{k, j}\right)\right]=0$ uniformly in $j$. Define $Q$ by

(d) $Q \equiv \bigcap_{k}\left(U_{i} I_{k, i}\right)$.

The continuous map $r: V_{i} \rightarrow Q$ will be the limit of a sequence of continuous maps $r_{k}: V_{i} \rightarrow Q$. To define $r_{k}$ choose points $p_{k, i} \in I_{k, i} \cap Q$, and for each $\left(x_{0}, x_{1}, \ldots\right) \in V$ set $r_{k}\left(x_{0}, x_{1}, \ldots\right)=p_{k, i}$ iff $x_{k} \in S_{k, i}$. The $r_{k}$ are well defined because the $S_{k, 1}$, $S_{k, 2}, \ldots$ are pairwise disjoint sets. Each $r_{k}$ is continuous because each $r_{k}^{-1}\left(p_{k, i}\right)$ is a closed subset of $V$. Moreover, if $x_{k} \in S_{k, i}, x_{k-1} \in S_{k-1, i^{\prime}}$, for $\left(x_{0}, x_{1}, \ldots\right)$ in $V$, then $h\left(S_{k, i}\right) \subset S_{k-1, i^{\prime}}$ because $h\left(x_{k}\right)=x_{k-1}$. So $r_{k}\left(x_{0}, x_{1}, \ldots\right)$ and $r_{k-1}\left(x_{0}, x_{1}, \ldots\right)$ are both contained in $I_{k-1, i^{\prime}}$ (see 2.6(a)). This assures that the $r_{k}$ converge to a continuous function $r: V \rightarrow Q$ (see 2.6(c)).

To show $\rho \times r: V_{i} \rightarrow\left[\Lambda \cap\left(B_{i}-\partial B_{i}\right)\right] \times Q$ is a homeomorphism it suffices to show that $\rho \times r$ is one-one and onto. Let $\left(x_{0}, x_{1}, \ldots\right),\left(z_{0}, z_{1}, \ldots\right)$ be points in $V$ which are mapped to the same point by $\rho \times r$. Then $x_{0}=z_{0}$, and, for all $k \geqslant 0, x_{k}$, $z_{k} \in S_{k, j}$ for some $j$ depending on $k$. Since $h\left(x_{k+1}\right)=x_{k}$ and $h\left(z_{k+1}\right)=z_{k}$, we have $x_{k}, z_{k} \in h\left(S_{k+1, j^{\prime}}\right)$ where $S_{k+1, j^{\prime}}$ contains $x_{k+1}, z_{k+1}$. It follows from 2.5(b), (c) that $h\left(S_{k+1, j}\right)$ is a smooth ball in $S_{k, j}$ and $h^{k}: h\left(S_{k+1, j^{\prime}}\right) \rightarrow K$ maps $h\left(S_{k+1, j^{\prime}}\right)$ diffeomorphically onto one of the smooth balls $B_{i}$ of 2.3(a). Since $h^{k}\left(x_{k}\right)=x_{0}=z_{0}=h^{k}\left(z_{k}\right)$, 
it follows that $x_{k}=z_{k}$ for all $k \geqslant 0$. This completes the verification that $\rho \times r$ is one-one.

To see that $\rho \times r$ is onto choose $x_{0} \times q \in\left[\Lambda \cap\left(B_{i}-\partial B_{i}\right)\right] \times Q$. Write $q=$ $\cap_{k} I_{k, j}$, where the $j$ 's depend on the $k$ 's. Using $2.5(\mathrm{~b})$, (c), choose points $x_{k} \in S_{k, j}$, $k \geqslant 1$, so that $h\left(x_{k}\right)=x_{k-1} \forall k \geqslant 1$ and the $j$ 's are the same as in the preceding intersection. Then $\left(x_{0}, x_{1}, x_{2}, \ldots\right)$ represents a point in $V_{i}$ such that $\rho \times$ $r\left(x_{0}, x_{1}, x_{2}, \ldots\right)=x_{0} \times q$. This completes the verification that $\rho \times r$ is onto.

Recall that a metric space $S$ is perfect if, for every $p \in S$, $\operatorname{closure}(S-p)=S$. If $S$ is compact and perfect then $S$ is homeomorphic to Cantor space (see [5]). The space $Q$ will be shown to be perfect, and hence homeomorphic to the Cantor set, if for every $I_{k, j}$ in 2.6 there are two distinct $I_{l, i}, I_{l, i^{\prime}}$ with $l>k$ and $I_{l, i} \cup I_{l, i^{\prime}} \subset I_{k, j}$. These intervals will exist iff there are distinct $S_{l, i}, S_{l, i^{\prime}}$ mapped into $S_{k, j}$ by $h^{l-k}$ (see 2.6(a)). The existence of $S_{l, i}, S_{l, i^{\prime}}$ follows from 1.4(d) and 2.5(b), (c).

This completes the proof of 2.1, modulo 2.5.

Proof of 2.5. It follows from 1.4(c), 2.2, and the compactness of $\Lambda$ that, for $\varepsilon$ sufficiently small and $l>0, h^{-l}(\bar{U})$ must be the union of a finite number of smooth balls $P_{k}$ so that each $P_{k}$ is mapped diffeomorphically onto one of the $B_{q}$ in 2.3(a), so 2.5 (a) is satisfied.

To verify $2.5(\mathrm{~b})$, consider any connected subset of $\bar{S}_{k, j}$ of the form $\cup_{i=1}^{N+1} P_{i}$. If $\varepsilon$ is chosen sufficiently small there will be an open neighborhood $U^{\prime}$ for $\cup_{i=1}^{N+1} P_{i}$ satisfying 2.3 (see 1.4(c) and 2.2). Applying 2.3(b) to both $\bar{U}, \bar{U}^{\prime}$, we see that $h\left(\cup_{i=1}^{N+1} P_{i}\right)$ is contained in a smooth ball $D$ and $h^{k}$ maps $h\left(\cup_{i=1}^{N+1} P_{i}\right)$ onto the smooth ball $h(\bar{U})$. Since $h\left(\cup_{i=1}^{N+1} P_{i}\right)$ is connected it follows that $h^{k}: h\left(\cup_{i=1}^{N+1} P_{i}\right) \rightarrow$ $h(\bar{U})$ must be a diffeomorphism. So $h\left(P_{i}\right)=h\left(P_{j}\right)$ for all $1 \leqslant i, j \leqslant N+1$, so $2.5(\mathrm{~b})$ must be true.

It follows from 2.3(c), as applied to $U^{\prime}$, that $P_{i}, P_{j} \subset B_{k}^{\prime}$ for some distinct $i, j$ and some $B_{k}^{\prime}$ as in 2.3(a). But then $h\left(P_{i}\right)=h\left(P_{j}\right)$ contradicts 2.3(b) unless $P_{i}=P_{j}$. It follows that there are at most $N$ distinct balls $P_{i}$ in $\bar{S}_{k, j}$. Letting $\bar{S}_{k, j}$ replace $\bar{U}$ and $\bar{P}_{i}$ replace $B_{i}$ in 2.3, it is seen from the above remark that $\bar{S}_{k, j}$ satisfies 2.3(a), (b). Let $Y$ be a smooth connected manifold in $\bar{S}_{k, j}$. Then, since $\bar{S}_{k, j} \subset \bar{U}^{\prime}, Y$ is contained in some $B_{k}^{\prime}$ (see 2.3(c) as applied to $\left.\bar{U}^{\prime}\right)$. Choose a ball $P$ in $B_{k}^{\prime}$ so that $h(P)=h\left(\bar{S}_{k, j}\right)$ and $P \supset Y$. Then $P$ must be one of the balls in the union $\cup_{i=1}^{N+1} P_{i}$, so $\bar{S}_{k, j}$ satisfies 2.3(c).

This completes the proof of 2.5 .

3. In this section the techniques developed by F. T. Farrell and the author [3] are used to construct the relative solenoids which yield the $\Lambda_{l}$ of 0.1 .

THEOREM 3.1. For each $l>0$ there is a relative l-solenoid $\Sigma\left(h_{l}\right)$ satisfying

(a) each $p \in \Sigma\left(h_{l}\right)$ has an open neighborhood $V$ in $\Sigma\left(h_{l}\right)$ which is homeomorphic to the product of the (zero-dimensional) Cantor set with a space $U$;

(b) $U$ is homeomorphic to an open subset of an l-dimensional Cantor set $C_{l}$.

The proof of 3.1 relies upon the following lemmas. Let $(M, \partial M)$ denote a compact differentiable manifold pair of dimension $m$, and $g:(M, \partial M) \rightarrow(M, \partial M)$ a continuous map covered by a bundle map $g^{*}:(T(M), T(\partial M)) \rightarrow(T(M), T(\partial M))$. 
LEMMA 3.2. There is a cell structure $D$ for $M$, containing $\partial M$ as a cell complex, and $a$ mapping $h:(M, \partial M) \rightarrow(M, \partial M)$, satisfying:

(a) the codimension one skeleton of $D, D^{m-1}$, is a smooth branched submanifold of $M$ meeting $\partial M$ transversely in a smooth branched submanifold of $\partial M$;

(b) $\left.h\right|_{D^{m-1}}$ is a smooth immersion $\left(D^{m-1}, \partial D^{m-1}\right) \rightarrow\left(D^{m-1}, \partial D^{m-1}\right)$, where $\partial D^{m-1} \equiv D^{m-1} \cap \partial M$, satisfying $1.4(\mathrm{e})$;

(c) there are $\lambda>1$ and a Riemannian metric $\|$ on the fibers of $T\left(D^{m-1}\right)$ so that $|d h(v)|>\lambda|v|$ for all nonzero $v \in T\left(D^{m-1}\right)$;

(d) $d h:\left(T\left(D^{m-1}\right), T\left(\partial D^{m-1}\right)\right) \rightarrow\left(T\left(D^{m-1}\right), T\left(\partial D^{m-1}\right)\right)$ is a (stably) fiber homotopic to $g^{*}:\left(\left.T(M)\right|_{D^{m-1}},\left.T(\partial M)\right|_{\partial D^{m-1}}\right) \rightarrow(T(M), T(\partial M))$.

For the next lemma, choose for each $(m-1)$-dimensional cell $G$ in $D^{m-1}$ a smooth $(m-1)$-dimensional ball $B_{G}$ in the interior of $G$. Let $E \subset D^{m-1}$ denote the complement of $\cup_{G} B_{G}^{0}$ in $D^{m-1}$, if $\partial M \neq \varnothing$, and set $E=D^{m-1}$ if $\partial M=\varnothing$. Note that $E$ is a smooth branched manifold with $\partial E=\left(\partial D^{m-1}\right) \cup\left(\cup_{G} \partial B_{G}\right)$, or $\partial E=$ $\partial D^{m-1}$ depending on whether $\partial M \neq \varnothing$ or $\partial M=\varnothing$.

LEMMA 3.3. There is a smooth immersion $\eta:(E, \partial E) \rightarrow\left(D^{m-1}, \partial D^{m-1}\right)$ satisfying the following:

(a) $\left.\eta\right|_{D^{m-2}}=$ identity, where $D^{m-2}$ is the $(m-2)$-skeleton of $D$.

(b) $\eta(G \cap E)=D^{m-1}$, for any $(m-1)$-dimensional cell $G$ of $D^{m-1}$.

Proof of Lemma 3.2. If $\partial M=\varnothing$, then 3.2 is an immediate corollary of Theorem 7.1 in [3]. The techniques of [3] can readily be adapted to prove 3.2 when $\partial M \neq \varnothing$ also.

Proof of Lemma 3.3. If $\partial M=\varnothing$ then $\eta: D^{m-1} \rightarrow D^{m-1}$ has already been constructed in $\S 8$ of [3] by techniques which can readily be adapted to construct $\eta$ : $E \rightarrow D^{m-1}$ even when $\partial E \neq \varnothing$.

Proof of TheOREM 3.1. The relative $l$-solenoid $\Sigma\left(h_{l}\right)$ is constructed from an immersion $h_{l}: W_{l} \rightarrow k_{l}$ (as in 1.4, 1.5 above) which will be described now.

In 3.2, 3.3, choose $M$ with $\operatorname{dim}(M)=l+2, \partial M \neq \varnothing$. Set

$$
K_{l} \equiv D^{m-1} \cup\left(\partial D^{m-1} \times[0,1]\right)
$$

where the union is taken along the identification $\partial D^{m-1} \cong \partial D^{m-1} \times 0$. Let $\partial E \times$ $[0, \varepsilon]$ be a one-sided collar for $\partial E$ in $K_{l}$, pointing out from $E$, so that in $\partial D^{m-1} \times[0,1]$ it agrees with $\partial D^{m-1} \times[0, \varepsilon]$. Set

$$
W_{l} \equiv E \cup(\partial E \times[0, \varepsilon]) .
$$

Define $\left.h_{l}\right|_{E}$ to be the composition

$$
\underbrace{\stackrel{\eta}{\rightarrow \rightarrow} D^{m-1} \stackrel{h^{k}}{\rightarrow} D^{m-1}}_{h_{l}}
$$

of the maps $\eta, h^{k}$ of 3.2, 3.3, where $k$ is chosen large enough to assure (by 3.2(c)) that $\left.h_{l}\right|_{E}$ is expansive. Now $\left.h_{l}\right|_{E}$ can be extended to $h_{l}: W_{l} \rightarrow K$, by the formula

$$
h_{l}(p, t) \equiv\left(h_{l}(p), g(t)\right) \in \partial D^{m-1} \times[0,1], \text { for any }(p, t) \in \partial E \times[0, \varepsilon],
$$


where $g:[0, \varepsilon] \rightarrow[0,1]$ is a suitable expansive map with $g(0)=0, g(\varepsilon)=1$. Clearly $\left.h_{l}\right|_{E},\left.h_{l}\right|_{\partial E \times[0, \varepsilon]}$ are both smooth expansive immersions. The half collar $\partial E \times[0, \varepsilon]$ can be chosen so that, for suitable $g$ in $3.5,\left.h_{l}\right|_{E}$ and $\left.h_{l}\right|_{\partial E \times[0, e]}$ together form a smooth immersion on all of $W_{l}$, which must also be expansive. Note $h_{l}\left(\partial W_{l}\right) \subset \partial K_{l}$, because $\partial W_{l}=\partial E \times \varepsilon$ and $\partial K_{l}=\partial D^{m-1} \times 1$. Note also that $h_{l}$ satisfies 1.4(e) because $h: D^{m-1} \rightarrow D^{m-1}$ does (3.2(b)). Finally we shall see that the map $h_{l}$ : $W_{l} \rightarrow K_{l}$ satisfies 1.4(d). Let $\Lambda \subset W_{l}$ be as in 1.4(d), $x \in \Lambda$, and $B$ a smooth $(l+1)$-dimensional ball in $W_{l}$ containing $x$ in its interior. We must show that under some power of $h_{l}$ a subset of $B$ is mapped onto $W_{l}$. To see this first note it follows from 3.3(b), the expansiveness of $h_{l}$, and the definition of $\left.h_{l}\right|_{E}$ given in 3.4, that any open set $U$ of $E$ contains a subset which is mapped onto $D^{m-1}$ under some power of $h_{l}$, and $h$ maps $\partial E \times[0, \varepsilon]-\partial D^{m-1} \times[0,1]$ onto $\partial D^{m-1} \times[0,1]$. Thus some power of $h_{l}$ maps a subset of $B$ onto $W_{l}$. On the other hand it follows from 3.5 that $\Lambda$ (of $1.4(d))$ lies in $E$. So any ball $B$ as in $1.4($ d) contains a subset $U$ which is an open subset of $E$. Thus $B$ contains a subset which is mapped onto $W_{l}$ under some power of $h_{l}$.

To complete the proof of 3.1 it remains to verify $3.1(\mathrm{a})$, (b) for the relative solenoid $\Sigma\left(h_{l}\right)$. By 2.1 and the fact $\Lambda \subset E$, it will suffice to show that $(B-\bar{\partial} B) \cap \Lambda$ is locally homeomorphic to an $l$-dimensional Cantor set, for any $(l+1)$-ball $B$ which is either smoothly embedded in $E-\partial E$ or which is smoothly embedded in $E$ and intersects $\partial E$ transversely in an $l$-ball, where $\bar{\partial} B=\partial B$ if $B \subset E-\partial E$, and $\bar{\partial} B$ $=\overline{\partial B-\partial E}$ if $B$ intersects $\partial E$ transversely. Consider the collection $\left\{B_{i}\right\}$ of smooth balls in $B-\partial B$ so that $B_{i}$ is mapped diffeomorphically onto one of the smooth balls $B_{G}$ (recall $\overline{D^{m-1}-E}=\cup_{G} B_{G}$ ), by some power of $h_{l}$. By using $3.2-3.5$, it can be deduced (left to the reader) that the following are true:

3.6.(a) $\left\{B_{i}\right\}$ is a disjoint collection of balls in $B-\partial B$.

(b) $\lim _{i \rightarrow \infty}\left[\operatorname{diameter}\left(B_{i}\right)\right]=0$.

(c) The closure of $\bigcup_{i} \partial B_{i}$ in $\overline{B-\cup_{i} B_{i}}$ equals $\overline{B-\cup_{i} B_{i}}$.

(d) For any $x \in \Lambda \cap(B-\bar{\partial} B)$ there is an open neighborhood $U$ for $x$ in $B-\bar{\partial} B$ so that $U \cap \Lambda=U \cap\left[\overline{B-\left(\cup_{i} B_{i}\right)}\right]$.

Now it follows from 3.6(a), (b), (c), that $\overline{B-\cup_{i} B_{i}}$ is an $l$-dimensional Cantor set $C_{l}$, so by $3.6(\mathrm{~d}),(B-\bar{\partial} B) \cap \Lambda$ is locally homeomorphic to $C_{l}$.

This completes the proof of 3.1 .

REMARK 3.7. In this remark I shall indicate how to obtain relative $l$-solenoids with even more complicated local topological types than the $\Sigma\left(h_{l}\right)$ of 3.1. These examples will be locally homeomorphic to the product of the (zero-dimensional) Cantor set with an l-dimensional Cantor type set, where by an l-dimensional Cantor type set is meant the closure (in $\left.B^{l+1}\right) \overline{B^{l+1}-\cup_{i} B_{i}}$, where the $B_{i}, i=1,2,3, \ldots$, are a collection of compact $(l+1)$-dimensional topological manifolds in $B^{l+1}$ satisfying

(a) $B_{i} \cap B_{j}=\varnothing$ if $i \neq j$;

(b) $\lim _{i \rightarrow \infty}\left[\right.$ diameter $\left.\left[B_{i}\right]\right]=0$;

(c) the closure of $\cup_{i} \partial B_{i}$ in $\overline{B^{l+1}-\cup_{i} B_{i}}$ equals $\overline{B^{l+1}-\cup_{i} B_{i}}$

Note $\overline{B^{l+1}-\cup_{i} B_{i}}$ is nowhere locally homeomorphic to an $l$-dimensional Cantor set if, for some $i>0$, all $B_{j}$ with $j \geqslant i$ are not $(l+1)$-balls. 
To get these more exotic relative $l$-solenoids we only need to modify $E$ in 3.3 . Recall that $\overline{D^{m-1}-E}$ is the union of $(m-1)$-balls $B_{G}$ (one $B_{G}$ in the interior of each $(m-1)$-cell $G$ of $D^{m-1}$ ). More generally, the techniques of $[3, \S 8]$ (to which we referred for the proof of 3.3) permit the construction of immersions $\eta:(E, \partial E) \rightarrow$ $\left(D^{m-1}, \partial D^{m-1}\right)$, satisfying the conclusion of 3.3 ., such that $\overline{D^{m-1}-E}$ is a union of $(m-1)$-dimensional compact manifolds $B_{G}$ (one in the interior of each $(m-1)$-cell $G$ of $D^{m-1}$ ) where we are allowed a wide latitude in the selection of the homotopy type of each $B_{G}$. Using such an $\eta:(E, \partial E) \rightarrow\left(D^{m-1}, \partial D^{m-1}\right)$, and defining $h_{l}$ : $\left(W_{l}, \partial W_{l}\right) \rightarrow\left(K_{l}, \partial K_{l}\right)$ as in 3.4 and 3.5 , yields the more exotic relative $l$-solenoids.

4. In this section the following will be proven:

THEOREM 4.1. The topological dimension of an l-dimensional Cantor set is equal to $l$.

Proof. It will suffice to show that the inclusion induced map $H_{l}(X) \rightarrow H_{l}\left(C_{l}\right)$ is injective for all closed subsets $X \subset C_{l}$, where $H_{*}()$ denotes the Čech-homology groups (see [6, Theorem VIII.3']). Set $M_{i}=B^{l+1}-\cup_{j=1}^{i} B_{i}$, where $B^{l+1}, B_{i}$ are as in 0.0 of the introduction. Note $M_{1} \supset M_{2} \supset M_{3} \supset \cdots$ and $C_{l}=\bigcap_{i} M_{i}$. Choose compact $(l+1)$-dimensional manifolds $N_{i} \subset M_{i}$ satisfying

4.1.(a) $N_{1} \supset N_{2} \supset N_{3} \supset \cdots$;

(b) $\cap_{i} N_{i}=X$;

(c) each inclusion $N_{i} \subset M_{i}$ induces an injective map $H_{l}\left(N_{i}\right) \rightarrow H_{l}\left(M_{i}\right)$.

The inclusion induced map $H_{l}(X) \rightarrow H_{l}(C)$ is the inverse limit of the following diagram of maps:

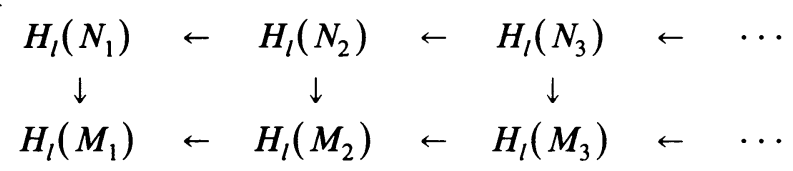

This inverse limit is injective by $4.1(\mathrm{c})$.

This completes the proof of 4.1 .

The concept of Cantor manifold is defined in [6, p. 93]. It seems likely that every $l$-dimensional Cantor set is an $l$-dimensional Cantor manifold.

Problem. Is every hyperbolic set locally the cartesian product of a zero-dimensional space with a Cantor manifold?

\section{BIBLIOGRAPHY}

1. S. Eilenberg and N. Steenrod, Foundations of algebraic topology, Princeton Univ. Press, Princeton, N.J., 1952.

2. F. T. Farrell and L. E. Jones, New attractors in hyperbolic dynamics, J. Differential Geom. 15 (1980), $107-133$.

3. __ Expanding immersions on branched manifolds, Amer. J. Math. 103 (1981), 41-101.

4. J. Guckenheimer, Endomorphisms of the Riemann sphere, Proc. Sympos. Pure Math., vol. 14, Amer. Math. Soc., Providence, R.I., 1970, pp. 95-123.

5. J. Hocking and G. Young, Topology, Addison-Wesley, Reading, Mass., 1961.

6. W. Hurewicz and H. Wallman, Dimension theory, Princeton Univ. Press, Princeton, N.J., 1948.

7. C. Robinson and R. Williams, Classification of expanding attractors: an example, Topology 15 (1976), 321-323.

8. S. Smale, Differential dynamical systems, Bull. Amer. Math. Soc. 73 (1967), 747-817.

9. R. Williams, Expanding attractors, Inst. Hautes Étude Sci. Publ. Math. 43 (1974), 169-203.

Department of Mathematics, State University of New York at Stony Brook, Stony Brook, NEW YORK 11794 